\title{
Combined virgin coconut oil and tocotrienol-rich fraction protects against bone loss in osteoporotic rat model
}

\author{
Mohd Maaruf Abdul Malik ${ }^{1,2}$, Faizah Othman ${ }^{1}$, Farida Hussan ${ }^{1,3}$, Ahmad Nazrun Shuid ${ }^{4}$ and Qodriyah Mohd Saad ${ }^{4}$ \\ 1. Department of Anatomy, Faculty of Medicine, Universiti Kebangsaan Malaysia Medical Centre, Jalan Yaacob Latif, \\ 56000 Cheras, Kuala Lumpur, Malaysia; 2. Centre of Preclinical Science Studies, Faculty of Dentistry, Universiti Teknologi \\ MARA, Sungai Buloh Campus, Jalan Hospital, 47000 Sungai Buloh, Selangor, Malaysia; 3. Department of Anatomy, \\ Human Biology Division, School of Medicine, International Medical University, Jalan Jalil Perkasa 19, Bukit Jalil, 57000 \\ Kuala Lumpur, Malaysia; 4. Department of Pharmacology, Faculty of Medicine, Universiti Kebangsaan Malaysia Medical \\ Centre, Jalan Yaacob Latif, 56000 Cheras, Kuala Lumpur, Malaysia. \\ Corresponding author: Mohd Maaruf Abdul Malik, e-mail: maaruf@uitm.edu.my \\ Co-authors: FO: faizahman@gmail.com, FH: khinpapah@gmail.com, ANS: anazrun@yahoo.com, \\ QMS: qodryrz@ymail.com \\ Received: 07-08-2019, Accepted: 29-10-2019, Published online: 25-12-2019
}

doi: www.doi.org/10.14202/vetworld.2019.2052-2060 How to cite this article: Malik MMA, Othman F, Hussan F, Shuid AN, Saad QM (2019) Combined virgin coconut oil and tocotrienol-rich fraction protects against bone loss in osteoporotic rat model, Veterinary World, 12(12): 2052-2060.

\begin{abstract}
Background and Aim: Both virgin coconut oil (VCO) and tocotrienol-rich fraction (TRF) are rich in antioxidants and may protect the bone against bone loss induced by ovariectomy and high-fat diet. The study aimed to determine the protective effects of combined therapy of VCO and TRF on osteoporosis in ovariectomized (OVX) rat fed with high-fat diet.

Materials and Methods: Thirty-six female Sprague-Dawley rats were divided into six groups: Sham-operated (SHAM), OVX control, OVX and given Premarin at $64.5 \mu \mathrm{g} / \mathrm{kg}(\mathrm{OVX}+\mathrm{E} 2)$, OVX and given VCO at $4.29 \mathrm{ml} / \mathrm{kg}(\mathrm{OVX}+\mathrm{V})$, OVX and given TRF at $30 \mathrm{mg} / \mathrm{kg}(\mathrm{OVX}+\mathrm{T})$, and OVX and given a combination of $\mathrm{VCO}$ at $4.29 \mathrm{ml} / \mathrm{kg}$ and TRF at $30 \mathrm{mg} / \mathrm{kg}$ $(\mathrm{OVX}+\mathrm{VT})$. Following 24 weeks of treatments, blood and femora samples were taken for analyses.

Results: There were no significant differences in serum osteocalcin levels between the groups ( $>0.05)$, while serum C-terminal telopeptide of Type I collagen levels of the OVX+VT group were significantly lower than the other groups $(\mathrm{p}<0.05)$. The dynamic bone histomorphometry analysis of the femur showed that the double-labeled surface/bone surface (dLS/BS), mineral apposition rate, and bone formation rate/BS of the OVX+E2, OVX+T, and OVX+VT groups were significantly higher than the rest of the groups $(\mathrm{p}<0.05)$.
\end{abstract}

Conclusion: A combination of VCO and TRF has the potential as a therapeutic agent to restore bone loss induced by ovariectomy and high-fat diet.

Keywords: bone loss, osteoporosis, ovariectomized rat, ovariectomy, tocotrienol-rich fraction, virgin coconut oil.

\section{Introduction}

Osteoporosis is known as a silent metabolic bone disease characterized by low bone mass and microarchitecture damage, resulting in increased risk of fractures. It is caused by reduced osteoblastic activity and increased osteoclastic activity. It commonly occurs in elderly women due to lack of estrogen following menopause [1]. Osteoporosis induced by estrogen deficiency could be further aggravated by unhealthy diet intake. The study has shown that cholesterol is one of the factors involved in stimulating osteoclast formation and survival [2] by promoting interleukin-1 production. Intake of repeatedly heated palm oil may be detrimental to the bone structure of ovariectomized (OVX) rat model [3]. A combination of these two unhealthy diets has been shown to worsen the bone

Copyright: Malik, et al. Open Access. This article is distributed under the terms of the Creative Commons Attribution 4.0 International License (http://creativecommons.org/licenses/by/4.0/), which permits unrestricted use, distribution, and reproduction in any medium, provided you give appropriate credit to the original author(s) and the source, provide a link to the Creative Commons license, and indicate if changes were made. The Creative Commons Public Domain Dedication waiver (http://creativecommons.org/ publicdomain/zero/1.0/) applies to the data made available in this article, unless otherwise stated. deterioration caused by ovariectomy [4]. The effects of the unhealthy diet were thought to be related to oxidative stress [5]. Estrogen has antioxidant effects and was positively correlated with the levels of plasma antioxidants and antioxidant enzymes [6]. Estrogen also enhanced the expression of glutathione peroxidase (GPX), an enzyme that degrades hydrogen peroxide [7]. Therefore, estrogen deficiency caused reduction in GPX, thus predisposing the bones to hydrogen peroxide. The lack of estrogen also reduces its protective effect against oxidative stress [8]. A previous study showed that estrogen deficiency stimulated bone loss, which in turn contributed to the development of osteoporosis [9].

Estrogen replacement therapy (ERT) is the primary treatment and prevention of postmenopausal osteoporosis [10]. Either estrogen alone or in combination with progesterone may prevent bone loss after menopause. These therapies are effective in preventing bone loss but do not reverse the bone loss. Longterm ERT may also increase the risk of breast cancer, coronary heart disease, stroke, and dementia [11]. Due to these serious side effects of ERT, researchers are finding alternative treatment [12] that is effective but 
has fewer side effects. One of the most popular natural products is virgin coconut oil (VCO), which has been extracted directly from fresh, mature coconut kernel without undergoing a refined process. This preserves the essential biologically active compounds in the oil such as tocotrienols, polyphenols, and tocopherols, which possess antioxidant properties [13]. Dietary supplementations of VCO had increased the antioxidant properties in rats [14]. VCO supplementation provided protection against bone loss in osteoporosis [15]. VCO has also been accounted to have anticancer, antimicrobial, and anti-inflammatory properties [16-18]. Furthermore, the effects of tocotrienols on bone parameters using different osteoporosis models such as ovariectomy [19], steroid-induced [20], and nicotine-induced [21] models have been conducted by researchers. All the studies showed that tocotrienols offered protection against bone loss in osteoporosis models. The mechanism of protection is related to its antioxidant properties.

Combined effects of VCO and tocotrienol-rich fraction (TRF) on bone loss in osteoporosis have not been explored yet and may be beneficial with the added oxidative stress of unhealthy diets. Therefore, the current study was designed to determine the effects of VCO and TRF, individually and in combination, on the bone parameters of OVX rat fed with high cholesterol diet and repeatedly heated palm oil.

\section{Materials and Methods}

\section{Ethical approval}

The research project was conducted from May 2014 to August 2016 in Universiti Kebangsaan Malaysia Medical Centre (UKMMC), Cheras, Kuala Lumpur, Malaysia. This research was approved by the Research and Ethical Committee, Faculty of Medicine, UKMMC (FP/ANAT/2014/FAIZAH).

\section{Experimental design}

Thirty-six female Sprague-Dawley rats, weighing between 250 and $300 \mathrm{~g}$, were obtained from the Laboratory Animals Resource Unit, Faculty of Medicine, UKMMC. The animals were allowed 1-week of acclimatization during which they were fed on commercial rat chow (Gold Coin, Klang, Selangor, Malaysia). The rats were randomized into six groups of six animals each: Sham-operated (SHAM), OVX-control, OVX and given Premarin $64.5 \mu \mathrm{g} / \mathrm{kg}(\mathrm{OVX}+\mathrm{E} 2), \mathrm{OVX}$ and given $\mathrm{VCO}$ $4.29 \mathrm{ml} / \mathrm{kg}(\mathrm{OVX}+\mathrm{V}), \mathrm{OVX}$ and given TRF $30 \mathrm{mg} / \mathrm{kg}$ $(\mathrm{OVX}+\mathrm{T})$, and $\mathrm{OVX}$ and given combination of VCO $4.29 \mathrm{ml} / \mathrm{kg}$ and TRF $30 \mathrm{mg} / \mathrm{kg}(\mathrm{OVX}+\mathrm{VT})$. The rats were housed one per cage in stainless-steel cages at $27 \pm 2^{\circ} \mathrm{C}$ with adequate ventilation and 12 -h light/dark cycle. After 2 weeks of ovariectomy, the SHAM group was fed with standard rat chow while the OVX rats were given high cholesterol diet mixed with repeatedly heated palm oil. All the rats were allowed free access to food and tap water ad libitum. The treatments were administered to the $\mathrm{OVX}+\mathrm{E} 2, \mathrm{OVX}+\mathrm{V}, \mathrm{OVX}+\mathrm{T}$, and OVX+VT groups daily through oral gavage for 24 weeks. The food intake and body weights were recorded daily and weekly, respectively. Blood was drawn to measure bone biochemical markers (osteocalcin $[\mathrm{OC}]$ and C-terminal telopeptide of Type I collagen [CTX]) in the serum before and after treatments. After 24 weeks, the rats were sacrificed, and the left femora were dissected and prepared for dynamic histomorphometric studies. All animal management and procedures were performed in accordance with the recommended guidelines for the care and use of laboratory animals. Care was taken to minimize discomfort, distress, and pain to the animals.

\section{Ovariectomy}

Ovariectomy was carried out under ketamil: ilium xylazil-20 (Troy Laboratories PTY, Australia) in 1:1 ratio, which was injected intramuscularly with the dose of $0.1 \mathrm{ml} / 100 \mathrm{~g}$ body weight of the rats. Once anesthetized, the furs on the abdomen were shaved. A vertical incision was made in the abdomen using a sterilized sharp knife, and both ovaries were identified. The fallopian tubes were ligated before removing the ovaries. The muscular layer under the skin was stitched up by catgut suture (Serafit, Germany) while the outer layer of skin was stitched with Mersilk Thread (Seralon, Serag Wiessner, Germany). The abdomen of the SHAM group was opened, whereby their ovaries were exposed and carefully manipulated but left intact [22]. The rats were left recuperating for 2 weeks before commencing the treatment.

\section{Preparation of high cholesterol diet and repeatedly heated palm oil}

Palm oil (Cap Buruh, Lam Soon Edible Oils, Kuala Lumpur, Malaysia) was purchased from a local manufacturer. The palm oil was heated 5 times. Briefly, $2.5 \mathrm{~L}$ of fresh palm oil was used to fry $1 \mathrm{~kg}$ of sweet potato slices in a stainless-steel wok. The temperature of the heated oil was maintained at $180^{\circ} \mathrm{C}$ for $10 \mathrm{~min}$. Then, the oil was cooled down at room temperature for $5 \mathrm{~h}$. The whole frying process was repeated 4 more times with a new batch of sweet potatoes without adding any fresh oil. Then, the 5 times heated oil $(5 \mathrm{HO})$ was collected to prepare the special diet by mixing $15 \%(\mathrm{w} / \mathrm{w})$ of $5 \mathrm{HO}$ to the high cholesterol diet. The diets were made into pellets and dried in the oven at $70^{\circ} \mathrm{C}$ overnight. The oil: high cholesterol diet ratio represents the average amount of daily oil intake in humans [23].

\section{Preparation of VCO, TRF, and Premarin}

The VCO used in this study was purchased from Bio-Asli Sdn. Bhd., Sungai Besar, Selangor, Malaysia. The VCO was administered through oral gavage using a cannula needle at a dose of $4.29 \mathrm{ml} / \mathrm{kg}$ body weight of rats for 24 weeks. The dose was equivalent to VCO given to humans for alternative therapy, which was three tablespoons or equal to $45 \mathrm{ml} /$ day [24].

TRF was prepared by Carotech (Tocomin, Selangor, Malaysia), consisting of alpha-tocotrienol 
(37.2\%), gamma-tocotrienol (39.1\%), and delta-tocotrienol $(22.6 \%)$. It was diluted in olive oil (Bertolli Classico, Italy) and given daily through oral gavages at the dose of $30 \mathrm{mg} / \mathrm{kg}$ body weight of rats for 24 weeks. This dose was roughly equivalent to $3 \mathrm{mg} / \mathrm{kg}$ in humans, or $210 \mathrm{mg}$ for a $70 \mathrm{~kg}$ man.

Estrogen dose given in this study was $64.5 \mu \mathrm{g} / \mathrm{kg}$. Each Premarin tablet containing $0.625 \mathrm{mg}$ of conjugated estrogens was crushed and dissolved in $20 \mathrm{ml}$ of distilled water. The solution was mixed using a magnetic stirrer until homogenous. Then, Premarin solution was stored in the refrigerator at $4^{\circ} \mathrm{C}$. The Premarin was given daily through oral gavage at the dose of $0.2 \mathrm{ml} / 100 \mathrm{~g}$ body weight of rats for 24 weeks.

\section{Blood collection and bone sampling}

For the biochemical study, blood samples were collected at the beginning and after 24 weeks of treatment from the retro-orbital vein under diethyl ether anesthesia. After leaving the blood at room temperature for $3 \mathrm{~h}$, the blood was centrifuged at $3000 \mathrm{rpm}$ for $10 \mathrm{~min}$ and the serum was stored at $-70^{\circ} \mathrm{C}$ until further use.

Following 24 weeks of treatment, the rats were anesthetized with diethyl ether and sacrificed humanely by cervical dislocation. The left femora were dissected, and adhering muscles were cleansed before fixing in $10 \%$ formalin.

\section{Biochemical markers parameter (OC and CTX)}

Bone biochemical markers of OC and CTX were evaluated before and after the treatment by enzyme-linked immunosorbent assay (ELISA's) machine (Leica CTR. MIC, Germany) using Rat-Mid OC ELISA kit (Nordic Biosciences, IDS, UK) and RatLaps $^{\mathrm{TM}}$ CTX-1 ELISA kit (Nordic Biosciences, IDS, UK), respectively.

Dynamic histomorphometric bone parameter
(single-labeled surface/bone surface [sLS/BS], dou-
ble-labeled surface/BS [dLS/BS], mineralized sur-
face/BS [MS/BS], mineral apposition rate [MAR],
and bone formation rate/BS [BFR/BS])

After fixation, the bones were cut sagittally at mid-shaft using a rotary electronic saw (Black \& Decker, USA). The distal left femora were then cut into half longitudinally and subsequently dehydrated in graded concentrations of ethanol. The femora were embedded in polymer methyl methacrylate medium according to the manufacturer's instructions (OsteoBed Bone Embedding Kit; Polysciences, USA). Then, the samples were sectioned at $7 \mu \mathrm{m}$ thickness using a Manual Rotary Microtome (Model 2235, Leica, Germany).

For dynamic parameters, undecalcified and unstained bones were analyzed using an image analyzer with Pro-Plus 5.0 software (Media Cybernetics, Silver Spring, MD, USA) that was connected to fluorescence microscope (Nikon Eclipse $80 \mu$, Japan). Dynamic parameters were measured using double fluorescent labeling technique by intraperitoneal injection of $20 \mathrm{mg} / \mathrm{kg}$ calcein to the rats at 7 days and 2 days before they were sacrificed. The basic measurements for dynamic parameters were sLS/BS, \%, $\mathrm{dLS} / \mathrm{BS}, \%, \mathrm{MS} / \mathrm{BS}, \%, \mathrm{MAR}, \mu \mathrm{m} /$ day, and BFR/BS, $\mu \mathrm{m}^{3} / \mu \mathrm{m}^{2} /$ day.

All dynamic measurements were carried out randomly at the metaphyseal region of the distal femora, which was located between $3 \mathrm{~mm}$ and $7 \mathrm{~mm}$ from the lowest point of the growth plate and $1 \mathrm{~mm}$ from the lateral cortex, excluding endocortical region [25]. The selected area was the secondary spongiosa area, which is rich in high-turnover trabecular bone. Trabecular bone was chosen because its remodeling process is more dynamic than cortical bone.

\section{Statistical analysis}

The Kolmogorov-Smirnov test was used as a normality test. The paired-sample t-test was carried out to compare the same group before and after treatment. For normally distributed data, one-way analysis of variance followed by Tukey's honestly significant difference post hoc test was utilized for comparison between treatment groups while Kruskal-Wallis and Mann-Whitney tests were used for data that were not normally distributed. Statistical analysis was performed using the Statistical Package for the Social Sciences software version 22.0 (SPSS Inc., Chicago, IL, USA). The results were presented as mean values \pm standard error of the mean. The statistical differences were considered significant at $\mathrm{p}<0.05$.

\section{Results}

\section{Mean daily food intake}

Figure-1 shows the mean daily food intake of all groups throughout the study treatment. The results showed that the OVX, OVX+E2, OVX $+\mathrm{V}$, and $\mathrm{OVX}+\mathrm{T}$ groups had significantly higher mean daily

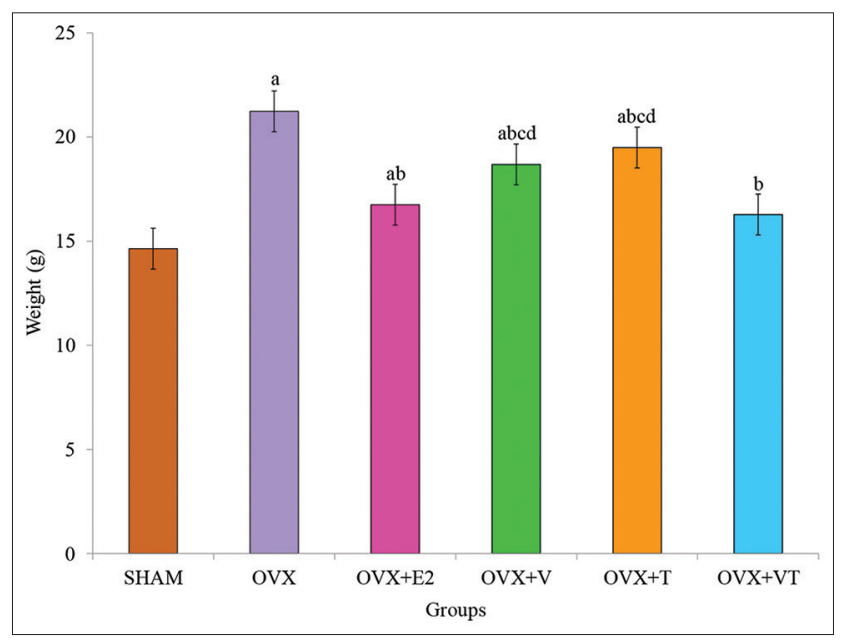

Figure-1: Mean daily food intake of all groups during the study treatment. Data are expressed as mean \pm standard error of the mean. (a) Significant difference compared to sham-operated group, (b) significant difference compared to ovariectomized group, (c) significant difference compared to ovariectomized with Premarin group, (d) significant difference compared to ovariectomized with virgin coconut oil and tocotrienol-rich fraction group. 
food intake compared to the SHAM group $(\mathrm{p}<0.05)$. All the OVX treated groups, OVX+E2, OVX $+\mathrm{V}$, $\mathrm{OVX}+\mathrm{T}$, and $\mathrm{OVX}+\mathrm{VT}$ groups, showed significant lower food consumption compared to the negative control, OVX group $(\mathrm{p}<0.05)$. Among the treated groups, both OVX+V and OVX $+\mathrm{T}$ groups had significantly higher mean daily food intake compared to the $\mathrm{OVX}+\mathrm{E} 2$ and $\mathrm{OVX}+\mathrm{VT}$ groups $(\mathrm{p}<0.05)$.

\section{Weight gain}

The body weight gain for all groups after 24-week study is shown in Figure-2. The body weight gain of the $\mathrm{OVX}, \mathrm{OVX}+\mathrm{V}, \mathrm{OVX}+\mathrm{T}$, and $\mathrm{OVX}+\mathrm{VT}$ groups was significantly higher than the control, SHAM group $(\mathrm{p}<0.05)$. The weight gain of the $\mathrm{OVX}+\mathrm{E} 2$ and $\mathrm{OVX}+\mathrm{T}$ groups was significantly lower than that of the negative control, OVX group $(\mathrm{p}<0.05)$. Among the treated groups, both $\mathrm{OVX}+\mathrm{V}$ and $\mathrm{OVX}+\mathrm{VT}$ groups had significantly higher body weight gain compared to the positive control, OVX+E2 group $(\mathrm{p}<0.05)$.

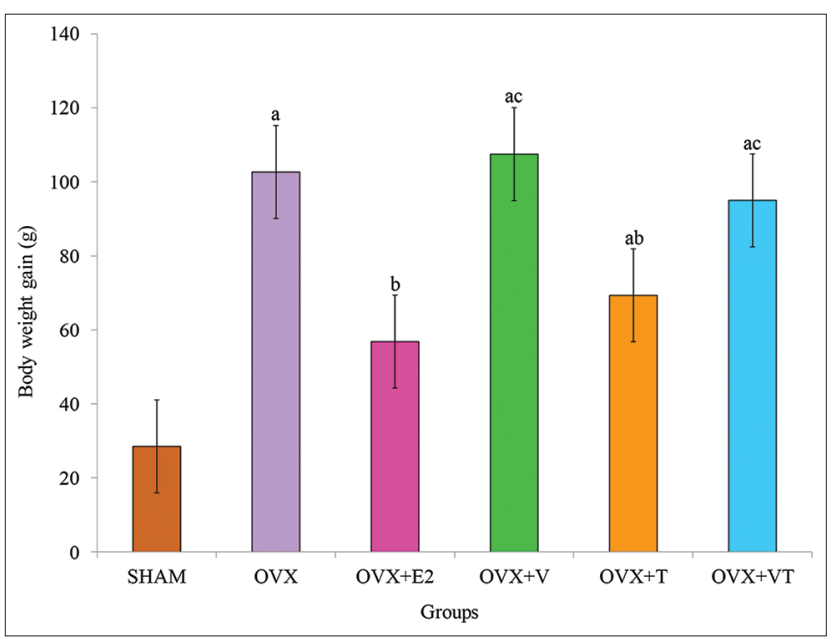

Figure-2: The body weight gain for all groups after 24-weeks study. Data presented as mean \pm standard error of the mean. (a) Significant difference compared to shamoperated group, (b) significant difference compared to ovariectomized group, (c) significant difference compared to ovariectomized with Premarin group.

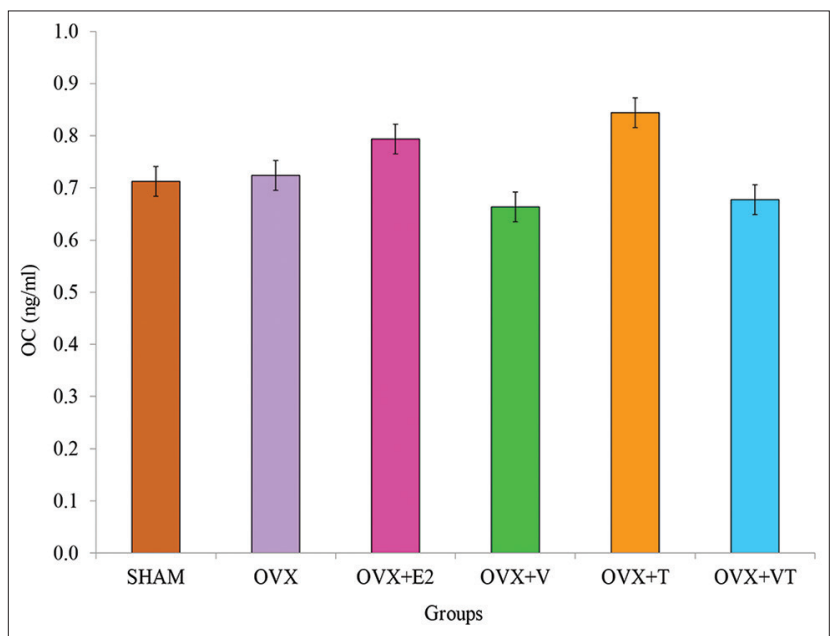

Figure-3: Post-treatment serum osteocalcin levels for all groups.

\section{Serum OC}

Figure-3 shows post-treatment serum OC levels for all groups. There was no significant difference in serum OC levels among the groups ( $\mathrm{p}>0.05)$.

\section{Serum CTX}

Figure- 4 shows post-treatment serum CTX levels for all groups. After 24 weeks of treatment, the serum CTX levels in the OVX+E2, OVX $+\mathrm{V}, \mathrm{OVX}+\mathrm{T}$, and OVX+VT groups were significantly lower compared to the control, SHAM group $(\mathrm{p}<0.05)$. Among the OVX groups, the post-treatment CTX level of the $\mathrm{OVX}+\mathrm{VT}$ group was significantly lower than the negative control, OVX group $(\mathrm{p}<0.05)$.

\section{SLS/BS}

The sLS/BS of the SHAM group was significantly higher compared to the OVX groups $(\mathrm{p}<0.05)$.

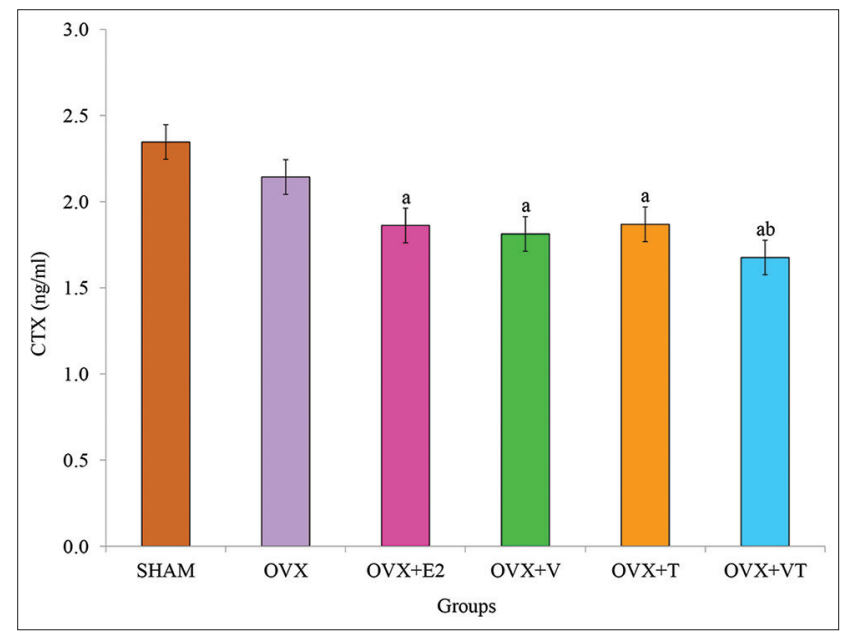

Figure-4: Post-treatment serum C-terminal telopeptide levels for all groups. (a) Significant difference compared to sham-operated group, (b) significant difference compared to ovariectomized group.

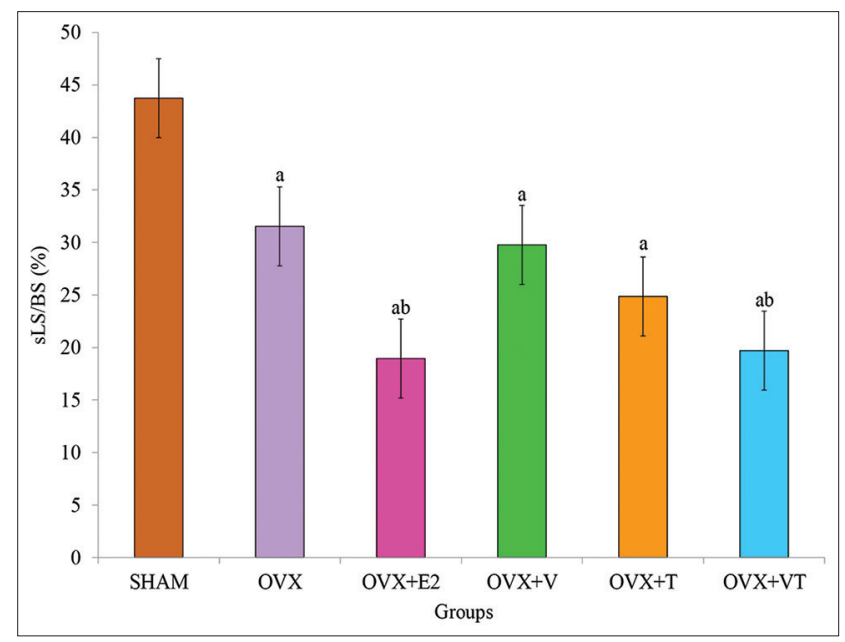

Figure-5: Single-labeled surface/bone surface. Data are expressed as mean \pm standard error of the mean. SHAM: Sham-operated; OVX: Ovariectomized; OVX+E2: Ovariectomized with Premarin; OVX+V: Ovariectomized with virgin coconut oil; OVX+T: Ovariectomized with tocotrienol-rich fraction; OVX+VT: Ovariectomized with virgin coconut oil and tocotrienol-rich fraction combination. (a) Significant difference compared to SHAM group, (b) significant difference compared to OVX group. 
The sLS/BS of the OVX+E2 and OVX+VT groups were significantly lower compared to the negative control, OVX group $(\mathrm{p}<0.05)$ (Figure-5).

\section{dLS/BS}

The dLS/BS of the OVX+E2, OVX+T and OVX + VT groups was significantly higher compared to the control, SHAM, and negative control, OVX groups $(\mathrm{p}<0.05)$ (Figure-6).

\section{MS/BS}

The MS/BS of the OVX+VT group was significantly higher compared to the control, SHAM group $(p<0.05)$. The MS/BS of the OVX+E2, OVX+T, and OVX+VT groups was significantly higher compared to the negative control, OVX group $(\mathrm{p}<0.05)$ (Figure-7).

MAR

The MAR of the OVX+E2, OVX+T, and OVX+VT groups was significantly higher compared to the control, SHAM, and negative control, OVX groups $(\mathrm{p}<0.05)$ (Figure-8).

\section{BFR/BS}

The OVX+E2, OVX+T, and OVX+VT groups had significant higher BFR/BS compared to the control, SHAM, and negative control, OVX groups $(\mathrm{p}<0.05)$ (Figure-9).

\section{Bone histology of dynamic parameter}

The photomicrographs of the trabecular bone of the distal part of femora were analyzed using fluorescence microscope. Both the control, SHAM and negative control, OVX groups showed an increase in sLS/BS than dLS/BS while the OVX+E2, OVX+V, $\mathrm{OVX}+\mathrm{T}$, and $\mathrm{OVX}+\mathrm{VT}$ groups had an increase in dLS/BS than sLS/BS (Figure-10).

\section{Discussion}

High cholesterol diet mixed with repeatedly heated palm oil was provided to the OVX rat model to mimic the unhealthy practice of postmenopausal women consuming diets high in cholesterol and containing repeatedly heated palm oil. These conditions would lead to oxidative stress, which may require extra antioxidant supplementations to help the body against free radical attacks [26]. In this study, two forms of well-known antioxidants, VCO and TRF, were used to protect the bone against free-radical induced damage.

Estrogen regulates food intake through the action of leptin, a protein that controls food intake [27]. Leptin level was decreased by ovariectomy [28], resulting in increased food consumption. Estrogen was directly involved in regulation of body weight by binding to estrogen receptors in subcutaneous fat tissue [29]. The result showed that the OVX group had significantly higher mean daily food intake and body weight gain compared to the rest of groups. This was in agreement with the previous reports that OVX rats have higher body weight gain due to fat deposition caused by deficiency of estrogen [30,31]. However, combined or individual supplementations of VCO and TRF to OVX rats were able to reduce the food intake pattern, until they were almost similar to the Premarin group (OVX+E2) and significantly lower than the OVX group. TRF given alone was able to control body weight gain which was comparable to Premarin. The findings on the effects of TRF in reducing body weight gain were similar to previous studies on OVX rats given estrogen [32] and calcium, estrogen, and TRF [33].

$\mathrm{OC}$, a bone formation marker, and CTX, a bone resorption marker, are bone biochemical markers

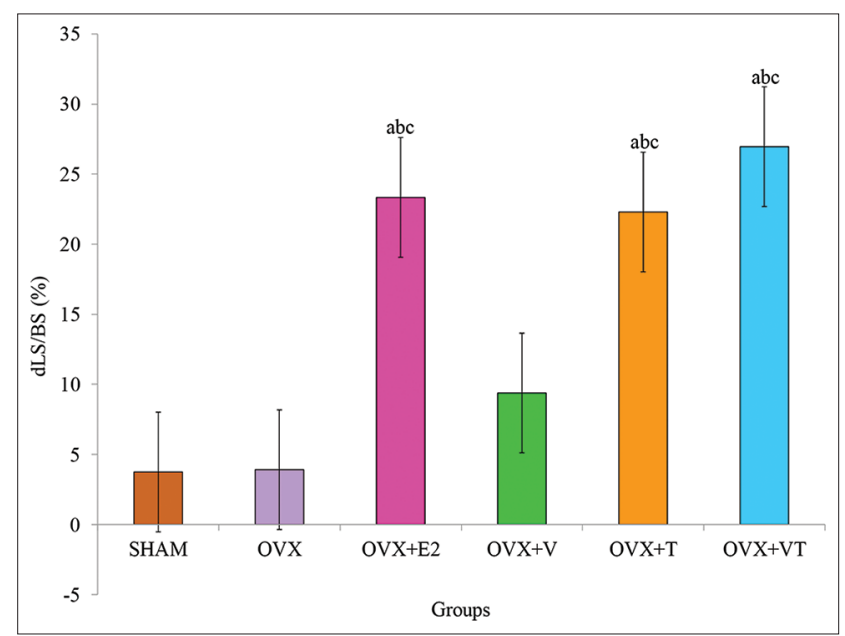

Figure-6: Double-labeled surface/bone surface. Data are expressed as mean \pm standard error of the mean. SHAM: Sham-operated; OVX: Ovariectomized; OVX+E2: Ovariectomized with Premarin; OVX+V: Ovariectomized with virgin coconut oil; OVX+T: Ovariectomized with tocotrienol-rich fraction; OVX+VT: Ovariectomized with virgin coconut oil and tocotrienol-rich fraction combination. (a) Significant difference compared to SHAM group, (b) significant difference compared to OVX group, (c) significant difference compared to $\mathrm{OVX}+\mathrm{V}$ group.

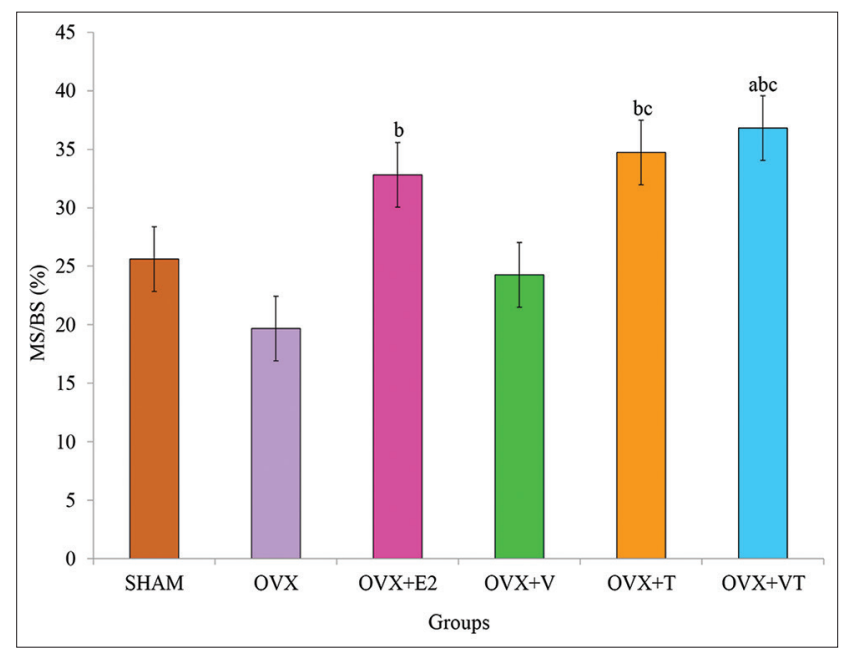

Figure-7: Mineralized surface/bone surface. Data are expressed as mean \pm standard error of the mean. SHAM: Sham-operated; OVX: Ovariectomized; OVX+E2: Ovariectomized with Premarin; OVX+V: Ovariectomized with virgin coconut oil; OVX+T: Ovariectomized with tocotrienol-rich fraction; OVX+VT: Ovariectomized with virgin coconut oil and tocotrienol-rich fraction combination. (a) Significant difference compared to SHAM group, (b) significant difference compared to OVX group, (c) significant difference compared to $\mathrm{OVX}+\mathrm{V}$ group. 


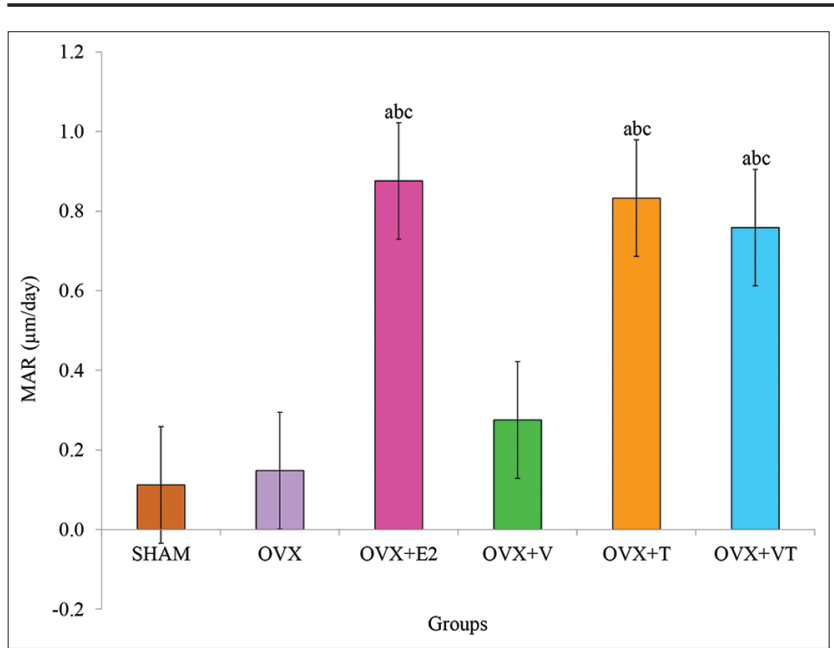

Figure-8: Mineral apposition rate. Data are expressed as mean \pm standard error of the mean. SHAM: Shamoperated; OVX: Ovariectomized; OVX+E2: Ovariectomized with Premarin; OVX+V: Ovariectomized with virgin coconut oil; OVX+T: Ovariectomized with tocotrienol-rich fraction; OVX+VT: Ovariectomized with virgin coconut oil and tocotrienol-rich fraction combination. (a) Significant difference compared to SHAM group, (b) significant difference compared to OVX group, (c) significant difference compared to OVX+V group.

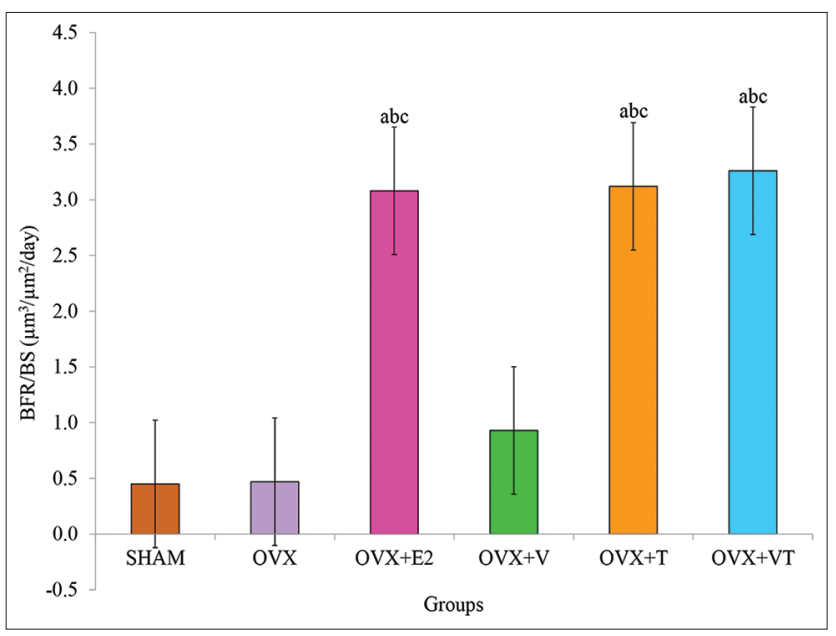

Figure-9: Bone formation rate/bone surface. Data are expressed as meantstandard error of the mean. SHAM: Sham-operated; OVX: Ovariectomized; OVX+E2: Ovariectomized with Premarin; OVX+V: Ovariectomized with virgin coconut oil; OVX+T: Ovariectomized with tocotrienol-rich fraction; OVX+VT: Ovariectomized with virgin coconut oil and tocotrienol-rich fraction combination. (a) Significant difference compared to SHAM group, (b) significant difference compared to OVX group, (c) significant difference compared to OVX+V group.

that could detect osteoblast and osteoclast activities, respectively [34,35]. In estrogen deficiency state, the increase in serum CTX levels was associated with the increase in bone turnover rate, leading to bone loss [36]. After 24 weeks of treatment, serum OC levels did not show any significant difference between the groups. This finding was in line with the previous study, which did not find any significant difference in the serum OC levels of groups supplemented with VCO or TRF after an 8-week period of treatment [37].
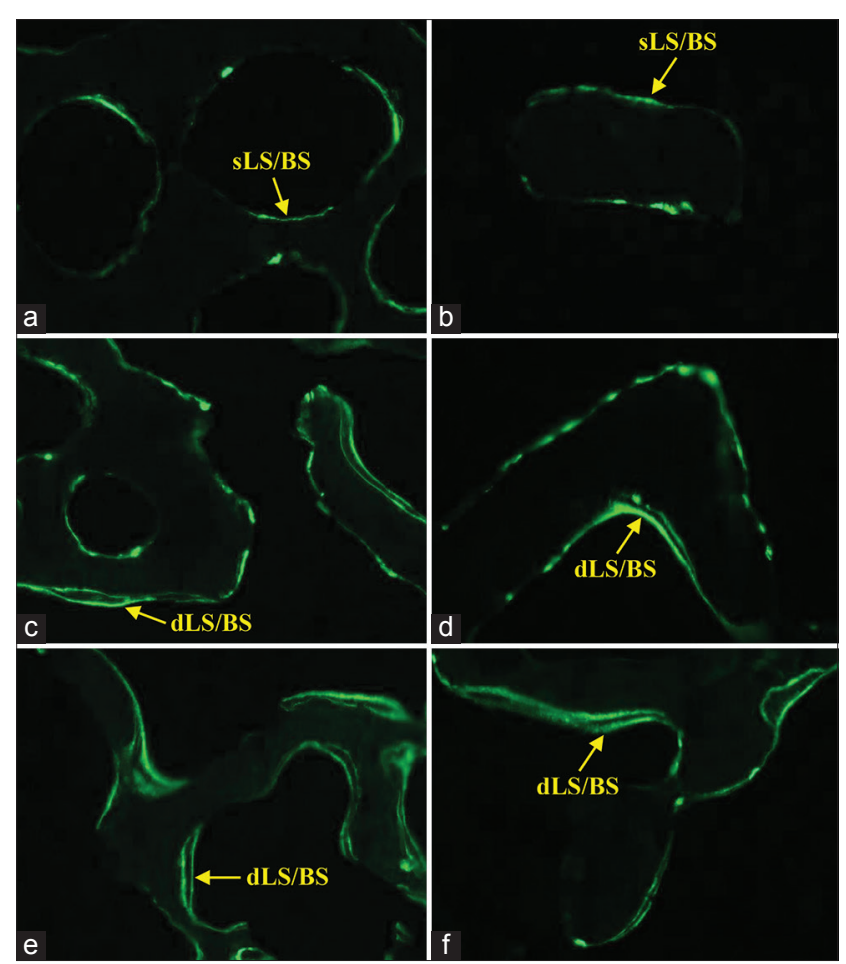

Figure-10: Photomicrographs of the trabecular bone of the distal part of femora labeled with calcein for bone histology of dynamic parameter using fluorescence microscope. Arrows indicated the single-labeled surface/bone surface and double-labeled surface/bone surface $20 \times$. (a) Shamoperated (SHAM), (b) ovariectomized-control (OVX), (c) ovariectomized treated with Premarin $(\mathrm{OVX}+\mathrm{E} 2)$, (d) ovariectomized treated with virgin coconut oil $(\mathrm{OVX}+\mathrm{V})$, (e) ovariectomized treated with tocotrienol-rich fraction $(\mathrm{OVX}+\mathrm{T})$, ( $\mathrm{f})$ ovariectomized treated with virgin coconut oil and tocotrienol-rich fraction combination (OVX+VT).

Hypercholesterolemia was positively correlated with reduction in bone formation and bone density, while bone resorption was increased [38]. The bone mineral density of OVX rats fed high cholesterol diet was significantly decreased after 7 months of treatment [39]. In this study, addition of cholesterol and repeatedly heated palm oil in the diet seemed to promote bone resorption. The result showed that the serum CTX levels were significantly lower in the $\mathrm{OVX}+\mathrm{E} 2, \mathrm{OVX}+\mathrm{V}, \mathrm{OVX}+\mathrm{T}$, and $\mathrm{OVX}+\mathrm{VT}$ groups compared to the SHAM group. This was in agreement with the previous study, which reported that treatment with anti-osteoporotic agents had decreased the serum CTX level [40]. The post-treatment CTX level in the OVX+VT group was also significantly lower compared to the OVX group. This indicated that VCO-TRF combination was able to reduce the raised CTX level by decreasing bone resorption and bone formation activities. The correlation between serum biomarkers with bone microarchitecture was demonstrated in laboratory animals and postmenopausal women studies [41,42].

Recent clinical diagnostic techniques for osteoporosis were mainly based on using of either X-rays or ultrasound. Both dual X-ray absorptiometry (DXA) and micro-computed tomography have become standard 
tools to evaluate bone mineral density and bone architecture, respectively. Among the most commonly used techniques, DXA was considered the current gold standard for osteoporosis diagnosis and fracture risk prognosis. However, as a research method, bone histomorphometry supported the interpretation of bone biology [43], developed the potential mechanism of actions of several effective therapies [44] and has been essential in identifying the adverse effects of drugs [45].

Thus, the dynamic bone histomorphometry parameter was used in this study and has been considered the ultimate histomorphometry assessment as it provides a quantitative assessment of the extent of bone formation over a specific period of time. The lower sLS/BS and the higher dLS/BS in both $\mathrm{OVX}+\mathrm{E} 2$ and $\mathrm{OVX}+\mathrm{VT}$ groups verified that the addition of Premarin and VCO-TRF supplementation had the potential to overcome the improper bone growth by stimulating bone formation as also seen in the MS/BS, MAR, and BFR/BS parameters. This indicated that VCO-TRF supplementation was as effective as Premarin in producing more newly mineralized bone. In addition, daily VCO-TRF supplementation had also increased the osteoblastic bone formation and decreased osteoclastic bone resorption in the OVX rats. This was indicated in the OVX+VT group by the higher dLS/BS, MS/BS, MAR, and BFR/ $B S$ values, and lower sLS/BS values compared to the OVX and OVX+V groups. Previous studies showed Vitamin E supplementation provided a positive effect on bone strength and bone mineral density in animal model studies [46-48].

Therefore, this study discovered that VCO-TRF supplementation on the OVX rats may have additive bone protective effects compared to single supplementation with VCO or TRF that can be beneficial in treating the postmenopausal osteoporosis. This study will help the researchers to uncover the critical areas of antioxidants combination that many researchers were not able to explore. Thus, a new theory on antioxidant therapy may be arrived at combined therapy of VCO and TRF.

\section{Conclusion}

The more superior osteoprotective effects of VCO-TRF supplementation indicated their worthiness as an alternative therapy for the prevention of postmenopausal osteoporosis. Further studies are required to explore their potential as an anti-osteoporotic agent for postmenopausal osteoporosis.

\section{Authors' Contributions}

FO conceived and designed the study. QMS provided research materials. FH provided logistic support. MMAM conducted research, collected, organized data, wrote the initial and final draft of the article. ANS analyzed, interpreted data, and checked the final draft of the article. All authors read and approved the final manuscript.

\section{Acknowledgments}

The authors expressed their gratitude to the Faculty of Medicine, Universiti Kebangsaan Malaysia, for their financial support by the Grant DLP-2013-005. Special thanks to Carotech Berhad (Malaysia) for supplying the palm tocotrienols. The authors also would like to thank Mr. Ahmad Tarmizi Abd. Razi, Ms. Siti Nor Ain Bakhtiar, Mr. Muhamad Arizi Aziz, Miss Nur Sabariah Adnan, and staff members of the Anatomy and Pharmacology Departments of Universiti Kebangsaan Malaysia Medical Centre for their technical assistance.

\section{Competing Interests}

The authors declare that they have no competing interests.

\section{Publisher's Note}

Veterinary World remains neutral with regard to jurisdictional claims in published institutional affiliation.

\section{References}

1. Riggs, B.L., Khosla, S. and Melton, L.J. (2002) Sex steroids and the construction and conservation of the adult skeleton. Endocr. Rev., 23(3): 279-302.

2. Xinyun, H., Yuan, L., Panpan, H., Zongbao, W., Fang, X., Linhao, H., Xilong, Z., Dawei, Z., Qi, C. and Chaoke, T. (2018) HDL impairs osteoclastogenesis and induces osteoclast apoptosis via upregulation of ABCG1 expression. Acta Biochim. Biophys. Sin., 50(9): 853-861.

3. Nazrun, A.S., Soelaiman, I., Yee, L.J., Loh, H.C., Yew, F.S., Mohamed, N., Gapor, T.M. and Jaarin, K. (2007) Reheating of soy oil is detrimental to bone metabolism in oestrogen deficient rats. Singapore Med. J., 48(3): 200-206.

4. Nazrun, A.S., Chew, C.M., Norazlina, M., Kamsiah, J. and Nirwana, I.S. (2007) The effects of repeatedly heated frying oil and high cholesterol diet on the bone in ovariectomized rats. Malays. J. Nutr., 13(1): 89-99.

5. Eloise, K., Chatelet, D.S., Johnston, D., Page, A., Tumbarello, D.A., Lotery, A. and Ratnayaka, J.A. (2019) Oxidative stress and dysfunctional intracellular traffic linked to an unhealthy diet results in impaired cargo transport in the Retinal Pigment Epithelium (RPE). Mol. Nutr. Food Res., 63(15): e1800951.

6. Sheng-Huang, C., Chieh-Hsin, C., Mu-Chun, Y., WenTung, H., Chia-Ying, H., Ya-Ting, H. and Jer-Yuh, L. (2015) Effects of estrogen on glutathione and catalase levels in human erythrocyte during menstrual cycle. Biomed. Rep., 3(2): 266-268.

7. Lean, J.M., Jagger, C.J., Kirstein, B., Fuller, K. and Chambers, T.J. (2005) Hydrogen peroxide is essential for estrogen-deficiency bone loss and osteoclast formation. Endocrinology, 146(2): 728-735.

8. Almeida, M., Han, L., Martin-Millan, M., Plotkin, L.I., Stewart, S.A., Roberson, P.K. Kousteni, S., O'Brien, C.A., Bellido, T., Parfitt, A.M., Weinstein, R.S., Jilka, RL. and Manolagas, S.C. (2007) Skeletal involution by age-associated oxidative. Stress and its acceleration by loss of sex steroids. J. Biol. Chem., 282(37): 27285-27297.

9. Carson, J.A. and Manolagas, S.C. (2015) Effects of sex steroids on bones and muscles: Similarities, parallels, and putative interactions in health and disease. Bone, 80(1): 67-78.

10. Ramírez, J., Nieto-González, J.C., Curbelo, R.R., Castañeda, S. and Carmona, L. (2017) Prevalence and risk factors for osteoporosis and fractures in axial 
spondyloarthritis: A systematic review and meta-analysis. Semin. Arthritis Rheum., 48(1): 44-52.

11. Rossini, M., Adami, S., Bertoldo, F., Diacinti, D., Gatti, D., Giannini, S., Giusti, A., Malavolta, N., Minisola, S., Osella, G., Pedrazzoni, M., Sinigaglia, L., Viapiana, O. and Isaia, G.C. (2016) Guidelines for the diagnosis, prevention and management of osteoporosis. Reumatismo, 68(1): 1-39.

12. Lim, D.W. and Kim, Y.T. (2013) Dried root of Rehmannia glutinosa prevents bone loss in ovariectomized rats. Molecules, 18(5): 5804-5813.

13. Yashi, S., Anil, D.S. and Gopal, K.S. (2018) Virgin coconut oil as functional oil. In: Therapeutic, Probiotic, and Unconventional Foods. Academic Press, Cambridge. p291-301.

14. Nevin, K.G. and Rajamohan, T. (2006) Virgin coconut oil supplemented diet increases the antioxidant status in rats. $J$. Clin. Food Chem., 99(2): 260-266.

15. Hayatullina, Z., Muhammad, N., Mohamed, N. and Soelaiman, I.N. (2012) Virgin coconut oil supplementation prevents bone loss in osteoporosis rat model. Evid. Based Complement. Altern. Med., 2012: 237236.

16. Ogbolu, D.O., Oni, A.A., Daini, O.A. and Oloko, A.P. (2007) In vitro antimicrobial properties of coconut oil on Candida species in Ibadan, Nigeria. J. Med. Food, 10(2): 384-387.

17. Hery, W., Hernayanti and Agus, P. (2008) Virgin coconut oil enriched with $\mathrm{Zn}$ as immune stimulate for vaginal candidiasis patient. J. Biosci., 15(4): 135-139.

18. Nevin, K.G. and Rajamohan, T. (2010) Effect of topical application of virgin coconut oil on skin components and antioxidant status during dermal wound healing in young rats. Skin Pharmacol. Physiol., 23(6): 290-297.

19. Norazlina, M., Ima-Nirwana, S., Gapor, M.T. and Khalid, B.A.K. (2000) Palm Vitamin E is comparable to $\alpha$-tocopherol in maintaining bone mineral density in ovariectomised female rats. Exp. Clin. Endocrinol. Diabetes., 108(4): 305-310.

20. Ima-Nirwana, S. and Fakhrurazi, H. (2002) Palm Vitamin E protects bone against dexmethasone induced osteoporosis in male rats. Med. J. Malaysia, 57(2): 136-144.

21. Norazlina, M., Lee, P.L., Lukman, H.I., Nazrun, A.S. and Ima-Nirwana, S. (2007) Effects of Vitamin E supplementation on bone metabolism in nicotine-treated rats. Singapore Med. J., 48(3): 195-199.

22. Al-Wahaibi, K., Al-Dhuhli, H., Diputado, T. and Alzadjali, N. (2008) Acute cardiovascular emergency: Missed killer in the emergency room. Oman Med. J., 23(2): 112-115.

23. Owu, D.U., Osim, E.E. and Ebong, P.E. (1998) Serum liver enzyme profile of Wistar rats following chronic consumption of fresh or oxidized palm oil diets. Acta Trop., 69(1): 65-73.

24. Daydrit, C.S. (2000) Coconut Oil in Health and Disease: It's and Monolaurin's Potential and Cure for HIV/AIDS. 25 July 2000. $37^{\text {th }}$ Cocotech Meeting, Chennai, India.

25. Baldock, P.A., Morris, H.A., Need, A.G., Moore, R.J. and Durbrige, T.C. (1998) Variation in the short-term changes in bone cell activity in three regions of the distal femur immediately following ovariectomy. J. Bone Miner. Res., 13(9): 1451-1457.

26. Kurutas, E.B. (2016) The importance of antioxidants which play a role in cellular response against oxidative/nitrosative stress: Current state. Nutr. J., 15(1): 71.

27. Zhu, L., Xu, P., Cao, X., Yang, Y., Hinton, A.O. Jr., Xia, Y. and $\mathrm{Xu}, \mathrm{Y}$. (2015) The ER $\alpha$-PI3K cascade in proopiomelanocortin progenitor neurons regulates feeding and glucose balance in female mice. Endocrinology, 156(12): 4474-4491.

28. Monteiro, R., Teixeira, D. and Calhau, C. (2014) Estrogen signaling in metabolic inflammation. Mediators Inflamm., 2014: 615917.
29. Davis, K.E., Carstens, E.J., Irani, B.G., Gent, L.M., Hahner, L.M. and Clegg, D.J. (2014) Sexually dimorphic role of $\mathrm{G}$ protein-coupled estrogen receptor (GPER) in modulating energy homeostasis. Horm. Behav., 66(1): 196-207.

30. Larco, D.O., Cruthirds, D.F., Weiser, M.J., Handa, R.J. and Wu, T.J. (2012) The effect of chronic immobilization stress on leptin signaling in the ovariectomized (OVX) rat. Endocrine, 42(3): 717-725.

31. Boldarine, V.T., Pedroso, A.P., Neto, N., Dornellas, A., Nascimento, C., Oyama, L.M. and Ribeiro, E.B. (2019) High-fat diet intake induces depressive-like behavior in ovariectomized rats. Sci. Rep., 9(1): 10551.

32. Shinoda, M., Latour, M.G. and Lavoie, J.M. (2002) Effects of physical training on body composition and organ weights in ovariectomized and hyperestrogenic rats. Int. J. Obes., 26(3): 335-343.

33. Norliza, M., Surayya, R., Ahmad, N.S., Norazlina, M. and Ima-Nirwana, S. (2013) Comparing the effects of tocotrienol-rich fraction, calcium and oestrogen on bone metabolism in ovariectomised rats. Sains Malays., 42(11): 1591-1597.

34. Zoch, M.L., Clemens, T.L. and Riddle, R.C. (2016) New insights into the biology of osteocalcin. Bone, 82: 42-49.

35. Liu, T.T., Liu, D.M., Xuan, Y., Zhao, L., Sun, L.H., Zhao, D.D., Wang, X.F., He, Y., Guo, X.Z., Du, R., Wang, J.Q., Liu, J.M., Zhao, H.Y. and Tao, B. (2017) The association between the baseline bone resorption marker CTX and incident dysglycemia after 4 years. Bone Res., 5: 17020.

36. Elwakf, A.M., Hassan, H.A. and Gharib, N.S. (2014) Osteoprotective effect of soybean and sesame oils in ovariectomized rats via estrogen-like mechanism. Cytotechnology, 66(2): 335-343.

37. Aktifanus, A.T., Shuid, A.N., Rashid, N.H.A., Ling, T.H., Loong, C.Y., Saat, N.M., Muhammad, N., Mohamed, N. and Soelaiman, I.N. (2012) Comparison of the effects of tocotrienol and estrogen on the bone markers and dynamic changes in postmenopausal osteoporosis rat model. Asian J. Anim. Vet. Adv., 7(3): 225-234.

38. Mandal, C.C. (2015) High cholesterol deteriorates bone health: New insights into molecular mechanisms. Front. Endocrinol., 6: 165.

39. Wang, T., Zhu, X., Dai, F., Li, C., Huang, D., Fang, Z. and Lu, Y. (2017) Effects of a standard high-fat diet with or without multiple deficiencies on bone parameters in ovariectomized mature rat. PloS One, 12(9): e0184983.

40. Yu-Te, Y., Jia-Hing, M., Bin, H., Chi-Yuan, M., ChenChen, Z., Teng, W.Y., Hong, J.Q., Li, S., Jiang, G.Y., Wang, W., Zhou, C.H. and Yan, S.G. (2018) A novel anti-osteoporotic agent that protects against postmenopausal bone loss by regulating bone formation and bone resorption. Life Sci., 209: 409-419.

41. Park, J.A., Ha, S.K., Kang, T.H., Oh, M.S., Cho, M.H., Lee, S.Y., Park, J.H. and Kim, S.Y. (2008) Protective effect of apigenin on ovariectomy-induced bone loss in rats. Life Sci., 82(25-26): 1217-1223.

42. Yi, D.W., Khang, A.R., Lee, H.W., Son, S.M. and Kang, Y.H. (2017) Association between serum cystatin C and bone mineral density in Korean adults. Ther. Clin. Risk Manage., 13: $1521-1528$

43. Khosla, S. (2013) Pathogenesis of age-related bone loss in humans. J. Gerontol. A Biol. Sci. Med. Sci., 68(10): 1226-1235.

44. Dempster, D.W., Roschger, P., Misof, B.M., Zhou, H., Paschalis, E.P., Alam, J., Ruff, V.A., Klaushofer, K. and Taylor, K.A. (2016) Differential effects of teriparatide and zoledronic acid on bone mineralization density distribution at 6 and 24 months in the SHOTZ study. J. Bone Miner. Res., 31(8): 1527-1535.

45. Odvina, C.V., Zerwekh, J.E., Rao, D.S., Maalouf, N., Gottschalk, F.A. and Pak, C.Y. (2005) Severely suppressed bone turnover: A potential complication of alendronate 
therapy. J. Clin. Endocrinol. Metab., 90(3): 1294-1301.

46. Ima-Nirwana, S., Wang, M., Roshayati, A.B., Hashnan, N.A., Ali, H.M., Mohamed, N., Muhammad, N. and Shuid, A.N. (2012) Palm tocotrienol supplementation enhanced bone formation in estrogen-deficient rats. Int. J. Endocrinol., 2012: 532862.

47. Chin, K. and Ima-Nirwana, S. (2014) The biological effects of tocotrienol on bone: A review on evidence from rodent models. Drug Des. Dev. Ther., 9: 2049-2061.

48. Zakaria, S., Mat-Husain, S.Z., Ying-Hwey, K., Xin-Kai, K., Mohd-Badawi, A., Abd-Ghani, N.A. and Mohamed, N. (2017) Vitamin E improved bone strength and bone minerals in male rats given alcohol. Iran. J. Basic Med. Sci., 20(12): 1360-1367.

$* * * * * * * *$ 